\title{
Evaluation of response characteristics of buried pipelines during earthquakes
}

\author{
K. Fuchida \\ Department of Architecture \& Civil Engineering, \\ Kumamoto National College of Technology, Kunamoto, Japan
}

\begin{abstract}
This paper presents an evaluating method for seismic behaviour of pipelines during earthquakes and studies the response characteristics of them for various conditions of ground displacements and pipelines. The proposed method consists of mainly two analytical methods which are the seismic response analysis of surface ground layers and the pseudo static response analysis of buried pipelines. The seismic response analysis method for surface ground layers is 2-dimensional effective stress analysis based on Biot's two phase mixture theory and Iai's constitutive equation. The pipeline analysis is based on a beam theory on an elastic foundation and modified transfer matrix method. Numerical computations are performed for various models with changing conditions of ground and pipes. As a result, axial and lateral response characteristics of pipelines are shown and evaluated by relating with earthquakes, grounds and pipelines conditions.

Keywords: response analysis, buried pipeline, surface layers, modified transfer matrix method.
\end{abstract}

\section{Introduction}

Severe damages of lifeline systems during earthquakes much affect the social activity and urban life of citizens. It is very important that aseismic investment for lifeline systems is performed in view of fast recovery of economical and social works in urban area after earthquakes.

This research aims to propose an evaluating method for seismic responses of buried pipelines and study the response characteristics of buried pipelines which are subjected to various seismic ground displacements with many conditions of pipelines and joints during earthquakes. 
The proposed method consists of two analytical methods. Firstly the responses of surface ground layers, in which a weak pipeline is buried, are investigated. Then detailed responses of pipelines are evaluated based on input soil displacements which are determined by referring to above responses of surface ground layers. The proposed method is briefly introduced as follows and some investigated examples of analyses are shown here.

\section{Evaluation method for pipeline responses}

The evaluating method for buried pipeline responses during earthquakes consists of two main analytical methods. The first method is the seismic response analysis for the surface ground layers. The analysis is performed by the existing computer program which is the effective stress analysis program "NUW2" [1-3]. The program "NUW2" is based on Biot's two phase mixture theory and Iai's constitutive equation [4]. The second method is the pseudo static response analysis for the buried pipeline. This method is based on the beam theory on an elastic foundation and modified transfer matrix method $[5,6]$. The flow of this synthetic method is shown in Figure 1.

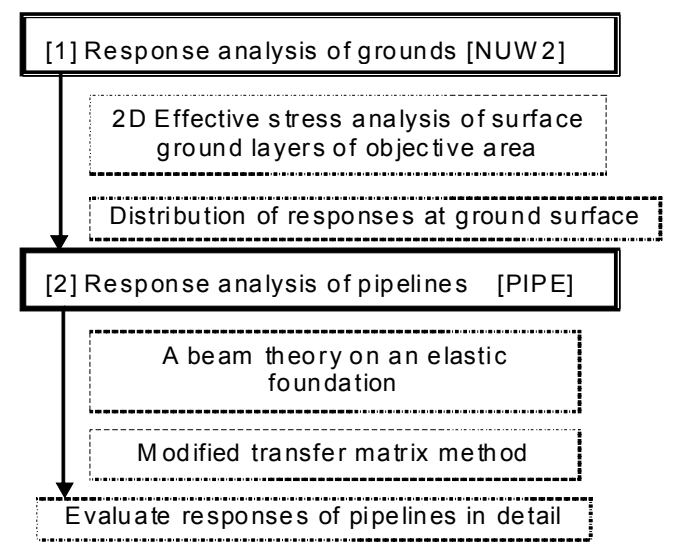

Figure 1: $\quad$ Flow of the evaluating method.

\subsection{Seismic response analysis for surface ground layers}

The analytical method for response of surface ground layers used in this study adopts the two-dimensional dynamic equilibrium equations for the soil-water phase and generalized Darcy law for the pore water based on Biot's two-phase mixture theory as in references [1-3]. To treat non-linearity (liquefaction) of the soil in the near field, the constitutive model for the plain condition is introduced. This constitutive model is constructed based on the 2-D strain-space multimechanism model for cyclic mobility of sandy soil first proposed by Iai et al. [4]. For the far field condition, the absorbing boundary condition is prepared. 
Above dynamic equilibrium equations are formulated to the finite element equation by considering the irreducible weak Galerkin formulation. The matrix form of finite element equation for a saturated porous medium with compressible pore water including the absorbing boundary condition may be written as:

$$
\begin{aligned}
& \left(\begin{array}{ll}
m_{u u} & m_{u w} \\
m_{u w}^{T} & m_{w w}
\end{array}\right)\left\{\begin{array}{l}
\ddot{\bar{u}} \\
\ddot{\bar{w}}
\end{array}\right\}+\left(\begin{array}{cc}
0 & 0 \\
0 & c_{w w}
\end{array}\right)\left\{\begin{array}{l}
\dot{\bar{u}} \\
\dot{\bar{w}}
\end{array}\right\}+\left(\begin{array}{cc}
K_{u u} & K_{u w} \\
K_{u w}^{T} & K_{w w}
\end{array}\right)\left\{\begin{array}{l}
\bar{u} \\
\bar{w}
\end{array}\right\}+\left\{\begin{array}{c}
\int_{\Omega} B_{u}^{T} \sigma^{\prime} d \Omega \\
0
\end{array}\right\} \\
& =\left\{\begin{array}{l}
\bar{f}_{u}+\bar{f}_{u}^{f} \\
\bar{f}_{w}+\bar{f}_{w}^{f}
\end{array}\right\}-\left(\begin{array}{cc}
c_{u u}^{\prime} & c_{u w}^{\prime} \\
c_{u w}^{\prime T} & c^{\prime}{ }_{w w}
\end{array}\right)\left\{\begin{array}{l}
\dot{\bar{u}}-\dot{\bar{u}}^{f} \\
\dot{\bar{w}}-\dot{\bar{w}}^{f}
\end{array}\right\}
\end{aligned}
$$

where $\mathrm{u}$ and $\mathrm{w}$ are the nodal displacement vectors and other notations can be seen in reference [2]. Equation (1) is coded as the 2D-FE effective stress analysis program of the name "NUW2".

\subsection{Response analysis for pipelines}

The responses of pipelines subjected to the ground displacement induced by liquefaction or seismic wave are analyzed by the program "PIPE" [5,6], which is based on the beam theory on an elastic foundation. Replacing the stiffness of liquefied or non-liquefied ground around the pipeline with a coefficient of subgrade reaction, the pipeline is modeled as pipeline-soil spring system as shown in Figure 2. The pipeline segments are connected by the axial and rotational joint spring $k_{\mathrm{t}}$ and $k_{r}$, respectively. The joint and soil springs are assumed to be bi-linearly elastic and the inertia and damping forces are neglected under the static load assumption. Based on these assumptions, when a ground motion is transmitted to a pipeline directly by soil spring, the governing axial and lateral equations of the pipeline are written by

a) axial direction

$$
-E A \frac{d^{2} u}{d x^{2}}+k_{s x} u=k_{s x} u_{s}
$$

b) lateral direction

$$
E I \frac{d^{4} v}{d x^{4}}+k_{s y} v=k_{s y} v_{s}
$$

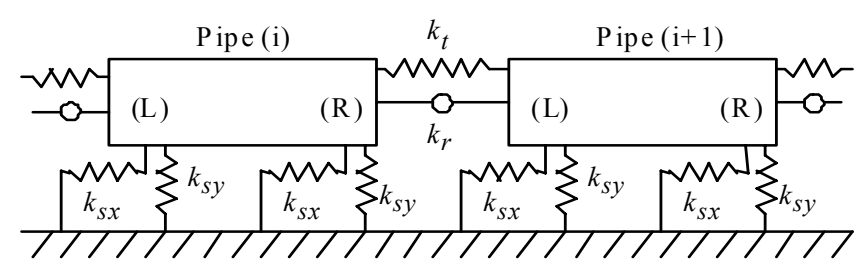

Figure 2: Modelling of pipeline-soil system. 
where $\mathrm{u}, \mathrm{v}=$ axial and lateral displacements of the pipeline respectively, $E, A$ and $I=$ Young's modulus, cross sectional area and geometrical moment of inertia of the pipeline respectively, $u_{\mathrm{s}}, v_{\mathrm{s}}=$ axial and lateral displacements of input ground motion respectively, $k_{\mathrm{sx}}, k_{\mathrm{sy}}=$ axial and lateral soil spring constants respectively.

In this study axial deformation of eq. (2) and bending deformation of eq. (3) are uncoupled. For prevention of numerical error accumulation, modified transfer matrix method is adopted. According to this method, the vector $y$ for the displacements and the vector $z$ for the forces are correlated as follows;

$$
\begin{aligned}
& \vec{a} y+\vec{b} z=\vec{c} \\
& \bar{a} y+\bar{b} z=\vec{c}
\end{aligned}
$$

where $a, b$ are square matrices, and the symbol $\rightarrow, \leftarrow$ mean the transfer from left to right and from right to left, respectively. Here the state vector $V$ at the pipeline element is defined as

$$
V=[y, z]
$$

where $y=[u, v, \phi], z=[N, M, Q], \phi, N, M, Q=$ deflection angle, axial force, bending moment and shear force at the end of pipeline segment. Then the eqs. (2) and (3) are also represented by the system equation as

$$
\frac{d V}{d x}=A V+f(x)
$$

where

$$
\begin{aligned}
& A=\left[\begin{array}{cccccc}
0 & 0 & 0 & -1 / E A & 0 & 0 \\
0 & 0 & -1 & 0 & 0 & 0 \\
0 & 0 & 0 & 0 & -1 / E I & 0 \\
-k_{s x} & 0 & 0 & 0 & 0 & 0 \\
0 & 0 & 0 & 0 & 0 & 1 \\
0 & -k_{s y} & 0 & 0 & 0 & 0
\end{array}\right], f(x)=\left[\begin{array}{c}
0 \\
0 \\
q(x) \\
0 \\
0 \\
p(x)
\end{array}\right] \\
& q(x)=k_{\mathrm{sx}} u_{\mathrm{s}}=\text { distributed axial load } \\
& p(x)=k_{\mathrm{sy}} v_{\mathrm{s}}=\text { distributed lateral load }
\end{aligned}
$$

A general solution of eq. (7) is given by

$$
V(x)=e^{A x} V(0)+e^{A x} \int_{0}^{x} e^{-A s} f(s) d s
$$

where $e^{A x}=$ transfer matrix.

The governing axial and lateral equations (2) and (3) are written as same as eq. (7);

$$
\frac{d}{d x}\left\{\begin{array}{c}
u \\
u^{\prime}
\end{array}\right\}=\left[\begin{array}{cc}
0 & 1 \\
\beta_{1}^{2} & 0
\end{array}\right]\left\{\begin{array}{l}
u \\
u^{\prime}
\end{array}\right\}+\left\{\begin{array}{l}
0 \\
-\beta_{1}^{2} u_{s}
\end{array}\right\}
$$




$$
\frac{d}{d x}\left\{\begin{array}{c}
v \\
v^{\prime} \\
v^{\prime \prime} \\
v^{\prime \prime \prime}
\end{array}\right\}=\left[\begin{array}{cccc}
0 & 1 & 0 & 0 \\
0 & 0 & 1 & 0 \\
0 & 0 & 0 & 1 \\
-4 \beta_{2}^{4} & 0 & 0 & 0
\end{array}\right]\left\{\begin{array}{c}
v \\
v^{\prime} \\
v^{\prime \prime} \\
v^{\prime \prime \prime}
\end{array}\right\}+\left\{\begin{array}{c}
0 \\
0 \\
0 \\
4 \beta_{2}^{4} v_{s}
\end{array}\right\}
$$

where $\beta_{1}=\sqrt{k_{s x} / E A}, \beta_{2}=\sqrt[4]{k_{s x} / E I}$, ' means the differential $d / d x$.

Using the vector $V$, the field transfer matrix $e^{A x}$ can be obtained. At the joint between the pipe segments $\mathrm{i}$ and $\mathrm{i}+1$, the following relations are obtained by considering the conditions of displacements and equilibrium;

$$
\begin{aligned}
& u_{i+1}^{L}=u_{i}^{R}-N_{i}^{R} / k_{T}, \quad v_{i+1}^{L}=v_{i}^{R}, \quad \phi_{i+1}^{L}=\phi_{i}^{R}-M_{i}^{R} / k_{R}, \\
& N_{i+1}^{L}=N_{i}^{R}, \quad M_{i+1}^{L}=M_{i}^{R}, \quad Q_{i+1}^{L}=Q_{i}^{R}
\end{aligned}
$$

where $k_{T}, k_{R}=$ spring constant of joint for translation and rotation, respectively.

\section{Results and considerations}

Numerical computations for the seismic response of surface ground layers are conducted for the Kobe ground models [3]. An example of surface layers model of Kobe ground by 2D-FE is shown in Figure 3. This cross section of the width of $2,250 \mathrm{~m}$ and the depth of $20 \mathrm{~m}$ to $30 \mathrm{~m}$ are divided into 100 and 6 finite elements, respectively. The ground models rest on the rigid base rock surface and have absorbing (viscous) boundaries (A. B.) at both sides. The left and right sides of the diagrams are toward mountain and sea sides, respectively. In the diagrams $\mathrm{N}$ denotes the standard penetration test $\mathrm{N}$-value which is converted to the initial soil stiffness (shear modulus) in the analysis. The geological parameters in Figure 5 are given as; A1 denotes the diluvial sandy gravel, A2 the alluvial sandy gravel, A3 the reclaimed soft soil, A5 the alluvial clay. Kobe earthquake recorded at GL-32m in Port Island is used as input seismic acceleration waves. The NS and UD components of this input waves are impinged simultaneously upward from horizontal base surface of the ground models with the maximum acceleration amplitude $A_{\max }$ of $0.1,1.0,5.4$ and $10.0 \mathrm{~m} / \mathrm{s}^{2}$. Figure 4 shows the distribution of maximum ground strain along cross section for four cases of the maximum acceleration amplitude $A_{\max }$. Large ground strain appears near the coastal areas where may refer to heavily damaged areas of pipelines.

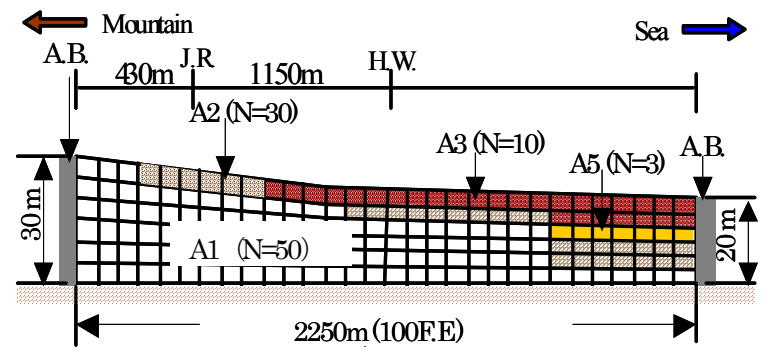

Figure 3: $\quad$ Example of surface ground layers model. 


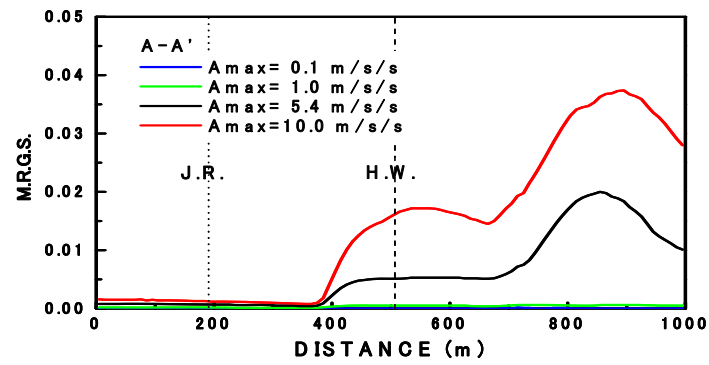

Figure 4: $\quad$ Maximum response ground strain near the surface (G.L.-2m).

Numerical computations are carried out for the responses of axial and lateral displacements and internal forces of pipelines. The input ground displacement is assumed to be uniformly distributed over the pipeline length. The reference pipeline dimensions are shown in Table 1. The reference soil spring characteristic is assumed to be represented by bi-linear coefficient of subgrade reaction as shown in Figure 5. The GM-type and S-type joint which resistant characteristic are shown in Figure 6 and 7, respectively, are used as the reference joints for segmented pipelines. The pipeline cases of the both ends fixed type and the cantilever type are evaluated subjected to lateral and longitudinal ground input, respectively.

Table 1: $\quad$ Reference pipe parameters.

\begin{tabular}{|c|c|}
\hline Physical items & Values(unit) \\
\hline Material of segment & Ductile cast iron \\
\hline Nominal diameter & $500(\mathrm{~mm})$ \\
\hline Thickness & $9.5(\mathrm{~mm})$ \\
\hline Total length & $100(\mathrm{~m})$ \\
\hline Young modulus & $1.57 \times 10^{8}\left(\mathrm{kN} / \mathrm{m}^{2}\right)$ \\
\hline Specific gravity & 7.15 \\
\hline Tensile strength & $3.92 \times 10^{5}\left(\mathrm{kN} / \mathrm{m}^{2}\right)$ \\
\hline Bending strength & $5.59 \times 10^{5}\left(\mathrm{kN} / \mathrm{m}^{2}\right)$ \\
\hline Allowable joint expansion & $50(\mathrm{~mm})$ \\
\hline Allowable joint rot. angle & $5($ degree$)$ \\
\hline
\end{tabular}

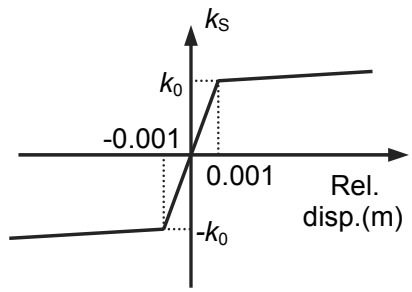

Figure 5: Characteristics of soil spring. 
Bending moment $(\mathrm{kN} \cdot \mathrm{m})$

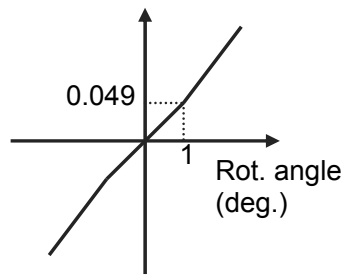

(a) Rotational joint spring
Axial force $(\mathrm{kN})$

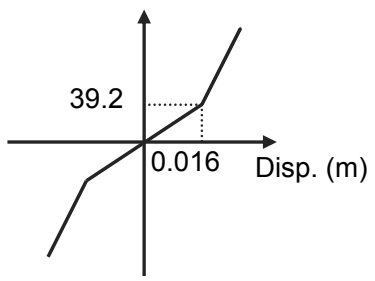

(b) Axial joint spring

Figure 6: Characteristics of GM-type joint.

Bending moment $(\mathrm{kN} \cdot \mathrm{m})$

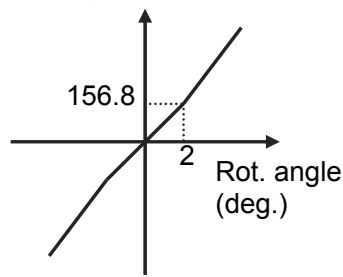

(a) Rotational joint spring

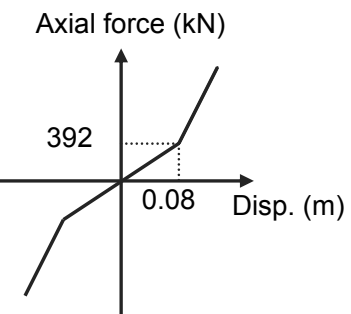

(b) Axial joint spring

Figure 7: Characteristics of S-type joint.

Figure 8 (a), (b) show the distribution of the responses of displacement and axial force of pipelines with S-type joint, respectively. Axial input soil displacement $0.01 \mathrm{~m}$ per unit length $1 \mathrm{~m}$ is almost accumulated at the joint as the expansion displacement. Maximum axial force $850 \mathrm{kN}$ which means maximum axial stress about $15600 \mathrm{kN} / \mathrm{m}^{2}$ is below tensile strength but maximum joint expansion $72.0 \mathrm{~mm}$ is larger than allowable one $50 \mathrm{~mm}$.

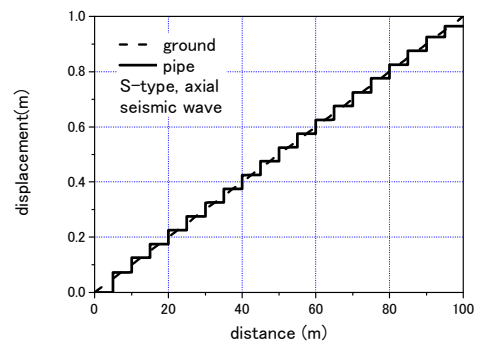

(a) Displacements

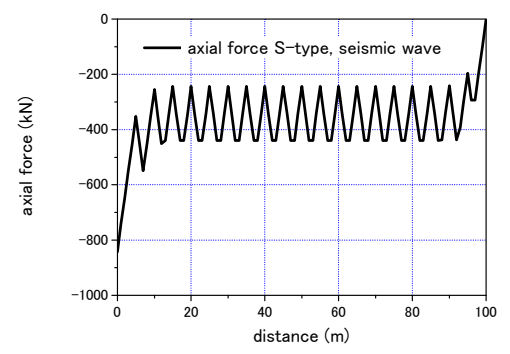

(b) Axial forces

Figure 8: Distribution of responses of pipelines. 
Figure 9 (a), (b) show the distribution of the responses of lateral displacement and bending moment of pipelines with S-type joint, respectively. Maximum lateral displacement of pipeline is $0.8 \mathrm{~m}$ as for $0.1 \mathrm{~m}$ input of soil. Maximum bending moment $2734 \mathrm{kNm}$ which means bending stress about $190000 \mathrm{kN} / \mathrm{m}^{2}$ is below tensile strength.

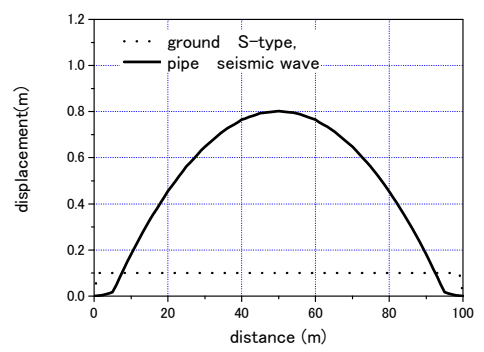

(a) Displacements

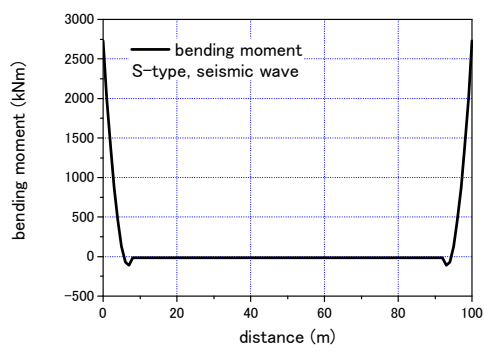

(b) Bending moments

Figure 9: Distribution of responses of pipelines.

Figure $10(a)$, (b) show the same distributions of responses as Figure 9. In Figure 10 the responses in the case of liquefaction are shown in which the pipeline with S-type joint is subjected to $5 \mathrm{~m}$ lateral flow displacement of soil. Maximum lateral displacement and bending moment of pipeline are about $1 \mathrm{~m}$ and $80 \mathrm{kNm}$, respectively. Maximum joint rotational angle reaches to about 1.6 degree which is less than the allowable value 5 degree. This means breakage of joint does not occur.

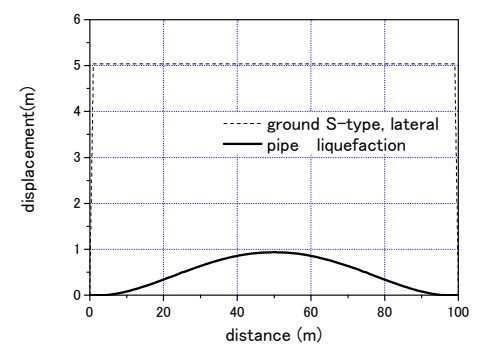

(a) Displacements

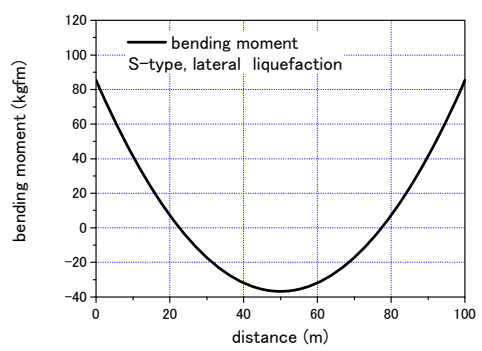

(b) Bending moments

Figure 10: Distribution of responses of pipelines.

Figure 11 (a), (b) show the same distributions of pipeline responses with GMtype joint as Figure 10. In Figure 11 the responses in the case of liquefaction are shown in which the pipeline is subjected to $5 \mathrm{~m}$ lateral flow displacement of soil. Maximum lateral displacement is about $9.6 \mathrm{~m}$, which is larger than that of pipeline with S-type joint because GM-type joint is more flexible than S-type 
one. In GM-type joint, maximum joint rotational angle for lateral input case reaches to about 19 degree which is larger than the allowable value 5 degree. This means breakage of joint.

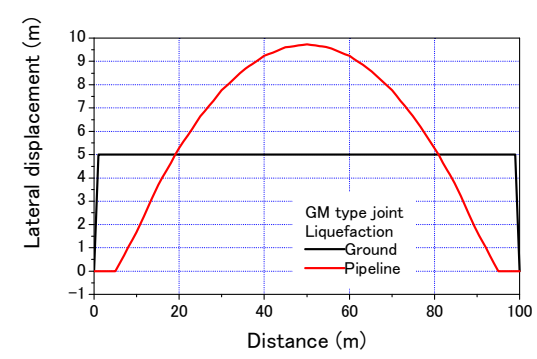

(a) Displacements

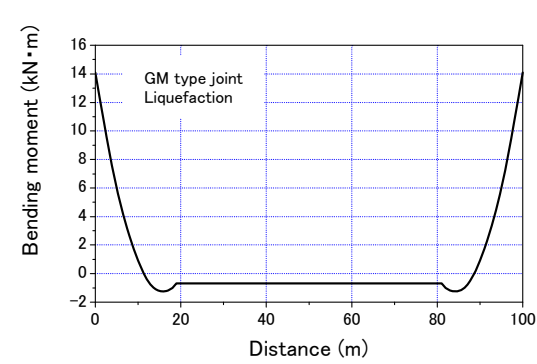

(b) Bending moments

Figure 11: Distribution of responses of pipelines (GM-type joint).

Figure 12(a), (b) show the distributions of responses of pipeline joint, in axial and lateral seismic wave input case, respectively. In Figure 12 the responses of joint expansions are shown in which the pipeline is subjected to $0.01 \mathrm{~m}$ axial and $0.1 \mathrm{~m}$ lateral displacement of soil, respectively. Maximum axial joint expansion in the case of axial input and lateral one are $72 \mathrm{~mm}$ and $113 \mathrm{~mm}$, respectively, which are over the allowable value $50 \mathrm{~mm}$.

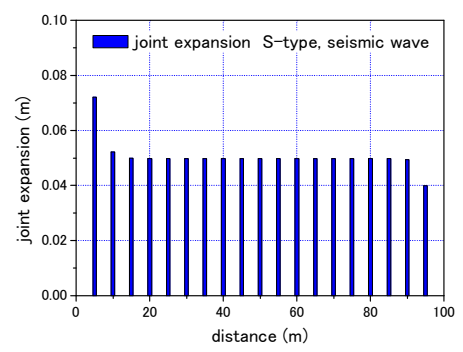

(a) Axial input

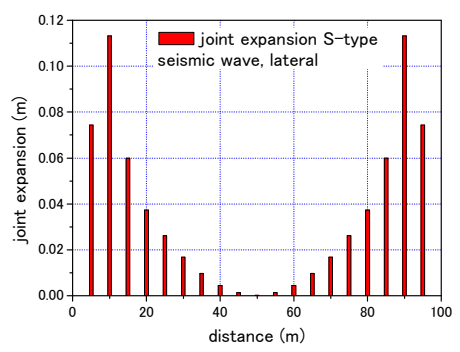

(b) Lateral input

Figure 12: Distribution of responses of pipeline joints.

Figure 13 shows the distributions of responses of joint rotational angle for lateral seismic wave input case. Maximum joint rotational angle reaches to about 1.6 degree which is smaller than the allowable value 5 degree. This means breakage of joint does not occur. 


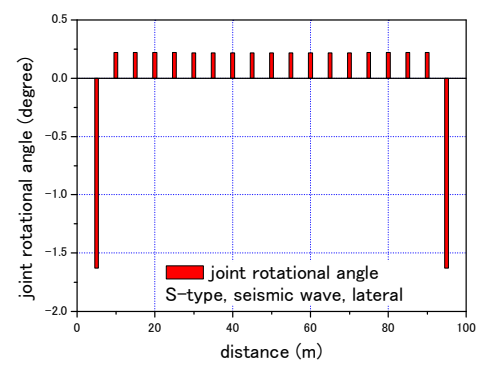

Figure 13: Distribution of responses of rotational angles.

\section{Conclusions}

In this study, the evaluation method for seismic responses of pipelines is proposed and response characteristics of pipelines are evaluated. The method consists of two different kinds of analyses which are the seismic response analysis of surface ground layers and the pseudo static response analysis of pipelines. Some numerical computational examples of pipeline responses are investigated. The proposed method is effective on evaluating the situation of whole conditions of pipelines and surface ground layers in perspective and the detailed pipeline responses including the response characteristics of surface ground layers.

\section{References}

[1] Akiyoshi, T., Fuchida, K. \& Fang, H. L., "Absorbing boundary conditions for dynamic analysis of fluid-saturated porous media", Soil Dynamics and Earthquake Engineering, 1994, 13(4), 387-397.

[2] Akiyoshi, T., Fuchida, K. et al., A Nonlinear Seismic Response Analysis Method for Saturated Soil-Structure System with Absorbing Boundary, Int. J. Numeric. and Analytic. Methods in Geomaech., 20(5), pp.307-329, 1996.

[3] Akiyoshi, T., Fuchida, K. \& Shirinashihama, S., Local site effects of transient dynamic characteristics of irregularly layered grounds, Proc. Fourth Int. Conf. on Recent Advances in Geotech. Earthq. Eng. And Soil Dynamics, Paper No.3.11, 2001.

[4] Iai, S. et al., Strain space plasticity model for cyclic mobility, Soils and Foundations, JSSMFE, Vol.32, No.2, pp.1-15, 1992.

[5] Fuchida, K., Wang, L.R.L. \& Akiyoshi, T., Parametric Analysis of Buried Pipelines Subjected to Liquefied Ground Movements, Proc. of 5th U.S. National Conference on Earthquake Engineering, pp.959-968, 1994.

[6] Fuchida, K., Akiyoshi, T., Sun, X., Preventive effect of ground improvement on liquefaction and destructive deformation of pipelines, Proc. of Third China-Japan-US Trilateral Symposium on Lifeline Earthquake Engineering, pp.141-148, 1998. 